\title{
Beta 2-microglobulin amyloidosis causing carpal tunnel syndrome, mimic steal syndrome
}

\begin{abstract}
Beta 2-microglobulin amyloidosis is a disabling condition that affects patients undergoing long-term renal dialysis. It's accumulation has been shown to invade synovial membranes and osteoarticular sites causing destructive osteoarthropathies, such as carpal tunnel syndrome (CTS). It involves organs like heart and tongue. This case report describes a 46-year-old Malaysian gentleman with hypertension and end stage renal failure for 14 years and on regular hemodialysis through a radiocephalic fistula. Recently, he developed bone and joints pain confined to the upper limb bilaterally, diagnosed as a renal hyper-parathyroidism. Total parathyroidectomy was done but symptoms not relieved. Pain struggle continued and the patient started to complain of bilateral hand numbness and decrease muscle power of the hands. Steal syndrome excluded and working diagnosis of CTS was made secondary to Beta 2-microglobulin amyloidosis after blood test conformation. Condition released surgically and patient regained his activity with no more pain.
\end{abstract}

Keywords: B2-micrglobulin amyloidosis, carpal tunnel syndrome, complication of hemodialysis
Volume 5 Issue 2 - 2018

\author{
Osama MH Mohamed,' Mustafa MTaher, ${ }^{2}$ \\ Walyeldin EM Elfakey ${ }^{3,4}$ \\ 'Department of Surgery, King Faisal Specialist Hospital and \\ Research Center, Saudi Arabia \\ ${ }^{2}$ Lecture and General Surgeon, Upper GI and Bariatric Division, \\ Malaysia National University, Malaysia \\ ${ }^{3}$ College of Medicine, University of Bahri, Sudan \\ ${ }^{4}$ Faculty of Medicine, Al-Baha University, Saudi Arabia
}

\begin{abstract}
Correspondence: Walyeldin EM Elfakey, Associate Professor, College of Medicine, University of Bahri, Khartoum, Sudan, Faculty of Medicine,Al-Baha University, Al Bahah, Saudi Arabia, Email walyeldin@aol.co.uk
\end{abstract}

Received: January 22, 2018 | Published: April 12, 2018

\section{Introduction}

Normal kidneys filter and remove excess small proteins from the blood; thus, keeping blood levels normal. When the kidneys failed, as in patients receiving dialysis, one type of small protein called beta 2-microglobulin builds up in the blood. When this occurs, beta 2-microglobulin molecules may join together, like the links of a chain, forming a few very large molecules from many smaller ones. These large molecules can form deposits and eventually damage the surrounding tissues and cause great discomfort. This condition is called dialysis-related amyloidosis (DRA). ${ }^{1}$

Table I Differences between CTS and steal syndrome

$\begin{array}{ll}\text { CTS } & \text { Hand pain more than } 8 \% \\ & \text { Radial pulse present } \\ \text { Intraneural median nerve hypervascularity (Doppler } \\ \text { detection) }\end{array}$

DRA is relatively common in patients, especially in older adults, who have been on hemodialysis (HD) for more than 5 years. HD membranes that have been used for many years don't effectively remove the large, complex beta 2-microglobulin proteins from the bloodstream. Newer HD membranes, as well as peritoneal dialysis, remove beta 2-microglobulin more effectively, but not enough to keep blood levels normal. As a result, beta 2-microglobulin blood levels remain elevated, and deposits form in bone, joints, and tendons. DRA may result in pain, stiffness, and fluid in the joints. Patients with DRA may also develop hollow cavities, or cysts, in some of their bones; these may lead to unexpected bone fractures. Amyloid deposits may cause tears in ligaments and tendons. ${ }^{2}$ Most patients with these problems can be helped by surgical intervention.

Half of the people with DRA also develop a carpal tunnel syndrome (CTS), which results from the unusual build up of protein in the wrists. Clinical manifestations almost never appear before 5 years of dialysis therapy. Unlike other types of amyloidosis, beta 2-microglobulin amyloid is confined largely to osteoarticular sites. ${ }^{3}$ Patients often present with a characteristic triad of CTS, shoulder pain, and flexor tenosynovitis in the hands. Visceral deposits are rare, occur after 10 or more years of dialysis, and tend to be symptomless in most cases. ${ }^{4}$

Bradish $^{5}$ reported eight cases of CTS in patients with HD, he concluded that precise cause has not been clearly established. However in our case report, we try to throw a light by proving one of the causes of this syndrome in patients on chronic HD.

No adequate treatment of beta 2-microglobulin amyloidosis exists. Medical therapy is limited to symptomatic approaches in ameliorating joint pain and inflammation.

The treatment of joint pain includes non-steroidal anti-inflammatory drugs, intra-articular injections of steroids, local steroid applicants, and low-dose oral systemic steroid in severe cases. Conservative treatment includes physical and occupational therapy. Wrist splints, cervical collars, lumbar corsets, knee braces, and immobilization for spondylo-arthropathies often are helpful. ${ }^{6}$

Surgical care of value in curing patient complain to certain extent but the risk of recurrence and failure is consider high as still the causative factor is there and re-accumulation of the protein deposit may recur the same symptoms again. Theoretically best treatment for B2-microglobulin amyloidosis is renal transplantation; however, not all the patients are fit for renal transplant. Usually the symptoms will 
disappeared after 1 week of the transplantation; however, the lytic bone lesions will never disappeared (Table 1). ${ }^{7}$

\section{Case report}

We are reporting a 46-year-old Malayan male. He is suffering from hypertension for 14 years. He is on regular follow-up for ESRF (endstage renal failure) secondary to hypertension more than 10 years on regular HD through a left radio-cephalic fistula.

Recently complained of joints and bones pain mainly at the lower limbs, sometimes complained muscle cramp and paresthesia more in the upper limbs and periphery. Further investigation revealed a hypercalcemia and diagnosis made as renal hyper-parathyroidism. Total parathyroidectomy was done; however, the patient's complain still there.

Pain and paresthesia continued on both hands, which were not related to the dialysis itself. Possibility of steal syndrome was ruled out because of: the fistula has good thrill, the radial pulse $1+$, doppler signal is biphasic over both radial and ulnar arteries and over the superficial palmar arch, doppler ultrasound showed a vein size of 1.8 $\mathrm{cm}$, the depth is $2 \mathrm{~mm}$, the flow is $1,200 \mathrm{ml} /$ minutes and no reversal of flow in the radial artery. So the diagnosis of steal syndrome has been excluded according to above mentioned findings.

Further investigation showed an elevated level of the beta 2-microglobulin protein that causing significant narrowing of the carpal tunnel retinaculum, so the diagnosis of CTS secondary to beta 2-microglobulin amyloidosis deposition in the wrist joint was made. The patient underwent surgical release of the carpal tunnel that resolved his complain.

\section{Discussion}

Amyloidosis is a condition characterized by the extracellular deposition of insoluble fibrillar proteins collectively termed amyloid (starch-like). Clinical manifestations almost never appear before 5 years of dialysis therapy. Unlike other types of amyloidosis, beta 2-microglobulin amyloid is confined largely to osteoarticular sites. Patients often present with a characteristic triad of CTS, shoulder pain, and flexor tenosynovitis in the hands. Visceral deposits are rare, occur after 10 years or more of dialysis, and tend to be symptomless in most cases.

The diagnosis of beta 2-microglobulin amyloidosis is established primarily by its clinical appearance on tissue or bone biopsy. The reference range of serum concentration of beta 2-microglobulin is $1.5-3 \mathrm{mg} / \mathrm{l}$. Serum levels of beta 2-microglobulin can be elevated to values of $50-100 \mathrm{mg} / 1$. Beta 2-microglobulin levels correlate with elevated serum creatinine levels and are inversely related to the glomerular filtration rate. ${ }^{5}$

\section{Radiographs}

Radiologic lesions typically present prior to the onset of pain. Joint erosions (usually involving large joints), lytic and cystic bone lesions, pathological fractures, spondyloarthropathies and vertebral compression fractures may be observed. However, conventional radiography may underestimate the extent of the disease ${ }^{6}$ Computerized tomography (CT) scans reveal amyloid deposits of intermediate attenuation. CT is the best method for detecting small areas of osteolysis in cortical bone or osseous erosion, and it may be helpful in the assessment of the distribution and extent of destructive changes. ${ }^{6}$ Magnetic resonance imaging have more accurate role than standard CT, ultrasound on the other hand has a rule in detecting the thickness of the tendon in cases of tenosynovitis. ${ }^{6}$

Treatment should be directed towards reduction of the beta 2-microglobulin burden of these patients, and recent studies showed that it can be achieved by modulation of the dialysis membranes used. However, medical therapy is limited to symptomatic approaches in ameliorating joint pain and inflammation. Medical therapy may range from conservative physiotherapy, anti-inflammatory medication, steroids whether local or systemic and may consider different membrane filter using in HD to achieve more clearance of the protein particles in patients with long standing ESRF. Surgical treatment offered as an option to those who benefit from surgical removal of the protein deposits in different parts of the body like in cases of CTS.

CTS as a major complication in HD patients and an increased incidence of CTS has been recently reported. While the rates vary considerably, it can be stated that CTS occurs in more than $10 \%$ of the patients under HD treatment over 10 years. ${ }^{7}$

Kachel et al. ${ }^{10}$ have observed amyloid deposits in 11 of 13 patients dialyzed for 8 years or more and operated on for CTS. Walts et al. ${ }^{11}$ have also reported high incidence of the amyloid deposits in synovia and tendon inside carpal tunnel in the patients with CTS. From these observation and from the literature before; there is a strong relation between patient of ESRF on regular HD and development of the CTS secondary to beta 2-microglobulin deposition which in our case patient present with signs of steal syndrome in certain extent but that was excluded from the clinical vascular evaluation for all the presence of pulsation and normal Doppler signal obtained over the distal circulation including the superficial palmar arch, and one more the patient complain was on both sides rather than fistula side (Figure 1).

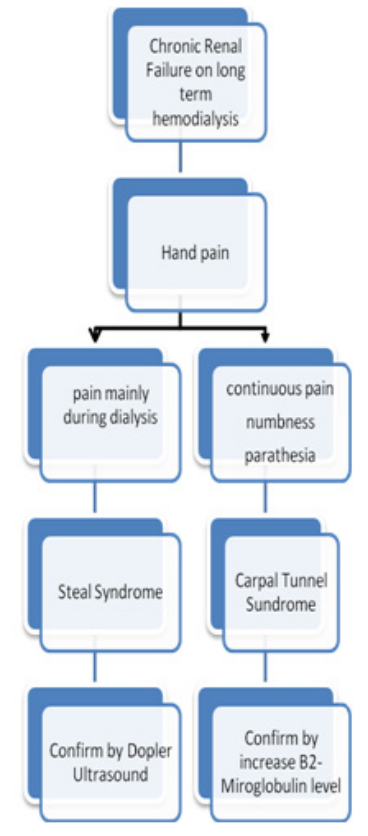

Figure I Suspicion and diagnosis of CTS and steal syndrome.

\section{Conclusion}

Patients with long standing ESRF on regular HD, due to poor kidney function may have insufficient protein wash-out during dialysis 
which lead to the building up beta 2-microglobulin into the synovial membrane of the wrist region that cause narrowing of the carpal tunnel which compress the passing tendons and nerves causing complain of pain, weakness, and numbness. These signs and symptoms to certain extent may show some similarity to steal syndrome secondary to poor distal blood supply secondary to reversal blood flow to the venous arm of the created fistula. Beta 2-microglobulin amyloidosis deposition in the writs joint causes CTS to mimic steal syndrome and proper clinical, biochemical, vascular, and radiological examination are mandatory to differentiate between these two conditions.

\section{Acknowledgements}

We thank all contributors to this work and specifically to the patient who accepted to be reported and the staff of Surgery Department at Malaysia National University Hospital.

\section{Conflict of interest}

We declare that there is no financial interest or any conflict of interest exists in this work.

\section{References}

1. Drueke T. Beta 2-microglobulin and amyloidosis. Nephrol Dial Transplant. 2000;15 Suppl 1:17-24.

2. Bely M, Kapp P, Szabo TS, et al. Electron microscopic characteristics of beta2-microglobulin amyloid deposits in long-term haemodialysis. Ultrastruct Pathol. 2005;29(6):483-491.

3. Kelly A, Apostle K, Sanders D, et al. Musculoskeletal pain in dialysisrelated amyloidosis. Can J Surg. 2007;50(4):305-306.

4. Yamamoto S, Kazama JJ, Maruyama H, et al. Patients undergoing dialysis therapy for 30 years or more survive with serious osteoarticular disorders. Clin Nephrol. 2008;70(6):496-502.

5. Bradish C. Carpal tunnel syndrome in patients on hemodialysis. $J$ Bone Joint Surg. 1985;67(1):130-132.

6. Kiss E, Keusch G, Zanetti M, et al. Dialysis-related amyloidosis revisited. AJR Am J Roentgenol. 2005;185(6):1460-147.

7. Charra B, Calemard E, Uzan M, et al. Carpal tunnel syndrome, shoulder pain and amyloid deposits in long term haemodialysis patients. Proc Eur Dial Transplant Assoc. 1985;21:291-295.

8. Ehler E. Median nerve ultrasonography in carpal tunnel syndrome. Clinical Neurophysiology Practice. 2017;2:186-187.

9. Delaney CP, Couse NF, Mehigan D, et al. Investigation and management of subclavian steal syndrome. Br J Surg. 1994;81(8):1093-1095.

10. Kachel HG, Altmeyer P, Baldamus CA, et al. Deposition of an amyloidlike substance as a possible complication of regular dialysis treatment. Contrib Nephrol. 1983;36:127-132.

11. Walts AE, Goodman MD, Matorin P. Amyloid, carpal tunnel syndrome, and chronic hemodialysis. Am J Neph. 1985;5(3):225-226. 